TRANSACTIONS OF THE

AMERICAN MATHEMATICAL SOCIETY

Volume 363, Number 7, July 2011, Pages 3439-3456

S 0002-9947(2011)05062-2

Article electronically published on February 8, 2011

\title{
CASTELNUOVO-MUMFORD REGULARITY OF EXT MODULES AND HOMOLOGICAL DEGREE
}

\author{
MARC CHARDIN, DAO THANH HA, AND LÊ TUÂN HOA
}

\begin{abstract}
Bounds for the Castelnuovo-Mumford regularity of Ext modules, over a polynomial ring over a field, are given in terms of the initial degrees, Castelnuovo-Mumford regularities and the number of generators of the two graded modules involved. These general bounds are refined in the case where the second module is the ring. Other estimates, for instance on the size of graded pieces of these modules, are given. We also derive a bound on the homological degree in terms of the Castelnuovo-Mumford regularity. This answers positively a question raised by Vasconcelos.
\end{abstract}

\section{INTRODUCTION}

Let $R$ be a polynomial ring in $n$ variables over a field and $M$ be a finitely generated graded $R$-module. Here we are interested in estimating several invariants of $M$ in terms of the degrees in a presentation of $M$ or in terms of the CastelnuovoMumford regularity of $M$.

The homological degree was introduced by Vasconcelos and his students ten years ago (see [DGV]). It has proved to be useful in many aspects (see, e.g., Chapter 9 in [Va] and $\mathrm{HHy2}$ ). One of our motivations was to answer positively a question of Vasconcelos [Va, page 261] on the existence of a polynomial bound on the homological degree of a module in terms of its regularity. In the case of a standard graded algebra $A$ of dimension $d>0$ with $n$ generators, our bound is

$$
\operatorname{hdeg}(A) \leq\left(\begin{array}{c}
\operatorname{reg}(A)+n \\
n
\end{array}\right)^{2^{(d-1)^{2}}} .
$$

We derive this bound from an estimate of the homological degree in terms of the Hilbert polynomial of a module. Namely, assume $M$ has dimension $d>0$, regularity $r$ and Hilbert polynomial $P$. Then

$$
\operatorname{hdeg}(M) \leq P(r)^{2^{(d-1)^{2}}}
$$

if $\operatorname{depth}(M)>0$ (the general case easily reduces to the result above).

Another new result concerns estimates on the size of the coefficients of the Hilbert polynomial of a module $M$ in terms of its regularity and the degree of its quotient

Received by the editors February 6, 2009.

2000 Mathematics Subject Classification. Primary 13D45.

Key words and phrases. Castelnuovo-Mumford regularity, local cohomology, canonical module, deficiency module, homological degree.

The second and third authors were supported in part by the National Basic Research Program (Vietnam). The third author would also like to thank University of Paris 6 for their financial support and hospitality during his visit in 2007 when this work was started.

(C)2011 American Mathematical Society 3439

Reverts to public domain 28 years from publication 
by $\operatorname{dim} M$ general linear forms. The bound in Theorem 4.6 refines and extends earlier results of the third author and is rather sharp.

Several estimates are necessary to obtain these results. One concerns the regularity of the modules $\operatorname{Ext}_{R}^{i}(M, R)$ in terms of the regularity and the Hilbert polynomial of $M$. This problem was first studied in $\underline{\mathrm{HaH}}$, and then continued in $\mathrm{HHy} 2$ for cyclic modules. It is an interesting problem because the regularity of the modules $\operatorname{Ext}_{R}^{i}(M, R)$ in some sense controls the behavior of the local cohomology module $H_{\mathfrak{m}}^{i}(M)$ in negative components. The bound found in [HHy2, Theorem 14] for cyclic modules is a huge number, and its proof required a rather complicated computation. Our bound here works for all modules and is much smaller; see Theorem 3.5. Its proof relies on the general estimates on $\operatorname{reg}\left(\operatorname{Ext}_{R}^{i}(M, N)\right)$ for a pair of modules proved in Section 2 together with considerations on the effect of the truncation of a module on its Ext's into $R$. An ingredient of the proof of Theorem 3.5 that may be of use elsewhere is Corollary 3.4, which expresses the Betti numbers of a module with a linear resolution in terms of the values of its Hilbert polynomial at some integers around the regularity.

Another type of estimate we establish concerns the vector space dimension of graded components of Ext modules. Besides the rather obvious estimates mentioned in Section 22 we prove more delicate bounds in Theorem 4.2 in terms of the Hilbert polynomial of the module and of its regularity. These can be used in turn to estimate, via graded local duality, the size of the graded components of local cohomology modules as the third author first did in [H, Theorem 3.4] for ideals. Our proof here is completely different from that in $[\mathrm{H}]$ : it is a direct proof, shorter and more elegant.

The general estimates on the regularity of $\operatorname{Ext}_{R}^{i}(M, N)$ for two graded modules $M$ and $N$ are proved in Section 2. We use the fact that these modules are homology modules of a complex of free modules whose shifts are controlled in terms of the ones appearing in free $R$-resolutions of $M$ and $N$. The regularity of the homology of a complex of free $R$-modules is estimated in terms of the regularities of cokernels of maps appearing in the complex, which are in turn bounded by the general results of Fall, Nagel and the first author in CFN]. In Section 3 we give a bound for the regularity of the Ext modules. Section 4 is devoted to the study of graded components of Ext modules and the Hilbert coefficients. In Section 5 we establish bounds for the homological degree of a module (see Theorem 5.2 and Theorem 5.5).

\section{General estimates on the Regularity of EXt modules}

Let $R$ be a polynomial ring in $n$ variables over a field with $n \geq 2$, equipped with its standard grading, and let $M$ and $N$ be finitely generated graded $R$-modules. We set end $(P):=\sup \left\{\mu \mid P_{\mu} \neq 0\right\}$ if $P \neq 0$ and end $(0)=-\infty$ and recall in the following definition the concepts of initial degree and regularity of a graded $R$-module.

Definition 2.1. Let $M$ be a graded $R$-module. Then $\operatorname{indeg}(M):=\inf \left\{\mu \mid M_{\mu} \neq 0\right\}$ if $M \neq 0$, indeg $(0):=+\infty$ and

$$
\operatorname{reg}(M):=\max _{i}\left\{\operatorname{end}\left(\operatorname{Tor}_{i}^{R}\left(M, R / R_{+}\right)\right)-i\right\}=\max _{i}\left\{\operatorname{end}\left(H_{R_{+}}^{i}(M)\right)-i\right\} .
$$

Let $H_{P}$ denote the Hilbert function of a graded $R$-module $P$.

We will estimate $\operatorname{reg}\left(\operatorname{Ext}_{R}^{i}(M, N)\right)$ in terms of the degrees appearing in free $R$ resolutions of $M$ and $N$. For doing so, we first give a bound on the regularity of 
the homology modules of a graded complex of free $R$-modules in terms of the shift that appears in it. Convention: $\left(\begin{array}{l}a \\ b\end{array}\right)=0$ if $a<b$.

Lemma 2.2. Let $F^{\bullet}$ be a graded complex of free $R$-modules with

$$
F^{i}:=\bigoplus_{f^{i} \leq j \leq b^{i}} R[-j]^{\beta_{i j}}
$$

Set $T^{i}:=\sum_{j} \beta_{i, j}$. Then, for any $i$,

(1) $\operatorname{indeg}\left(H^{i}\left(F^{\bullet}\right)\right) \geq \operatorname{indeg}\left(F^{i}\right)=f^{i}$,

$\operatorname{reg}\left(H^{i}\left(F^{\bullet}\right)\right) \leq \max \left\{b^{i}, b^{i+1},\left[T^{i+1}\left(b^{i}-f^{i+1}\right)\right]^{2^{n-2}}+f^{i+1}+2,\left[T^{i}\left(b^{i-1}-f^{i}\right)\right]^{2^{n-2}}+f^{i}\right\}$,

(3) for any $\mu \geq f^{i}$,

$$
\operatorname{dim}_{k}\left(\left(H^{i}\left(F^{\bullet}\right)\right)_{\mu} \leq \operatorname{dim}_{k}\left(F^{i}\right)_{\mu} \leq T^{i}\left(\begin{array}{c}
\mu-f^{i}+n-1 \\
n-1
\end{array}\right),\right.
$$

(4) for any $j$ and any $\mu \geq f^{i}+j$,

$$
\operatorname{dim}_{k}\left(\operatorname{Tor}_{j}^{R}\left(H^{i}\left(F^{\bullet}\right), k\right)\right)_{\mu} \leq T^{i}\left(\begin{array}{c}
n \\
j
\end{array}\right)\left(\begin{array}{c}
\mu-f^{i}-j+n-1 \\
n-1
\end{array}\right) .
$$

Proof. Statements (1) and (3) are obvious, and (4) follows from (3) and from the fact that $k$ is resolved as an $R$-module by the Koszul complex on the variables of $R$ (see the proof of Lemma 2.5).

We now prove statement $(2)$. Set $H^{i}:=H^{i}\left(F^{\bullet}\right)$. The exact sequences

$$
\begin{gathered}
0 \rightarrow \operatorname{im}\left(d^{i-1}\right) \rightarrow \operatorname{ker}\left(d^{i}\right) \rightarrow H^{i} \rightarrow 0, \\
0 \rightarrow \operatorname{ker}\left(d^{i}\right) \rightarrow F^{i} \rightarrow F^{i+1} \rightarrow \operatorname{coker}\left(d^{i}\right) \rightarrow 0
\end{gathered}
$$

and $0 \rightarrow \operatorname{im}\left(d^{i-1}\right) \rightarrow F^{i} \rightarrow \operatorname{coker}\left(d^{i-1}\right) \rightarrow 0$ imply the estimates

$$
\begin{aligned}
\operatorname{reg}\left(H_{i}\right) & \leq \max \left\{\operatorname{reg}\left(\operatorname{ker}\left(d_{i}\right)\right), \operatorname{reg}\left(\operatorname{im}\left(d^{i-1}\right)\right)-1\right\} \\
& \leq \max \left\{\operatorname{reg}\left(F^{i}\right), \operatorname{reg}\left(F^{i+1}\right)+1, \operatorname{reg}\left(\operatorname{coker}\left(d^{i}\right)\right)+2, \operatorname{reg}\left(\operatorname{coker}\left(d^{i-1}\right)\right)\right\} .
\end{aligned}
$$

By [CFN, Theorem 3.5], the presentation $F^{j} \rightarrow F^{j+1} \rightarrow \operatorname{coker}\left(d^{j}\right) \rightarrow 0$ shows that, for any $j$,

$$
\operatorname{reg}\left(\operatorname{coker}\left(d^{j}\right)\right) \leq\left[T^{j+1}\left(b^{j}-f^{j+1}\right)\right]^{2^{n-2}}+f^{j+1} .
$$

The inequality in (2) follows.

Corollary 2.3. Let $F^{\bullet}$ be a graded complex of free $R$-modules with $F^{i}:=R[r+i]^{T_{i}}$. Then:

(1) $\operatorname{indeg}\left(H^{i}\left(F^{\bullet}\right)\right) \geq \operatorname{indeg}\left(F^{i}\right)=-r-i$,

$$
\operatorname{reg}\left(H^{i}\left(F^{\bullet}\right)\right) \leq \max \left\{T_{i+1}^{2^{n-2}}+1, T_{i}^{2^{n-2}}\right\}-r-i,
$$

(3) for any $\mu \geq-r-i$,

$$
\operatorname{dim}_{k}\left(\left(H^{i}\left(F^{\bullet}\right)\right)_{\mu} \leq \operatorname{dim}_{k}\left(F^{i}\right)_{\mu} \leq T_{i}\left(\begin{array}{c}
\mu+r+i+n-1 \\
n-1
\end{array}\right),\right.
$$

(4) for any $j$ and any $\mu \geq-r-i+j$,

$$
\operatorname{dim}_{k}\left(\operatorname{Tor}_{j}^{R}\left(H^{i}\left(F^{\bullet}\right), k\right)\right)_{\mu} \leq T_{i}\left(\begin{array}{c}
n \\
j
\end{array}\right)\left(\begin{array}{c}
\mu+r+i-j+n-1 \\
n-1
\end{array}\right) .
$$


For a finitely generated graded $R$-module $P$, set $T_{i}^{P}:=\operatorname{dim}_{k} \operatorname{Tor}_{i}^{R}(P, k), f_{i}^{P}:=$ $\operatorname{indeg}\left(\operatorname{Tor}_{i}^{R}(P, k)\right)$ and $b_{i}^{P}:=\operatorname{reg}\left(\operatorname{Tor}_{i}^{R}(P, k)\right)$ (recall that indeg $(0)=+\infty$ and $\operatorname{reg}(0)=-\infty)$.

Theorem 2.4. Let $M$ and $N$ be finitely generated graded modules over the polynomial ring $R$. With notation as above, set $T^{i}=\sum_{p-q=i} T_{p}^{M} T_{q}^{N}, r_{M}:=\operatorname{reg}(M)-$ $\operatorname{indeg}(M), r_{N}:=\operatorname{reg}(N)-\operatorname{indeg}(N)$ and $\vdots=\operatorname{indeg}(M)-\operatorname{indeg}(N)$. Then, for any $i$,

(1) $\operatorname{indeg}\left(\operatorname{Ext}_{R}^{i}(M, N)\right) \geq e_{i}:=\operatorname{indeg}(N)-\operatorname{reg}(M)-i$, and equality holds for some $i$,

(2)

$$
\operatorname{reg}\left(\operatorname{Ext}_{R}^{i}(M, N)\right)+i \leq\left(r_{M}+r_{N}+1\right)^{2^{n-2}} \max \left\{T^{i}, T^{i+1}\right\}^{2^{n-2}}+1-,
$$

(3) for any $\mu \geq e_{i}$,

$$
\operatorname{dim}_{k}\left(\operatorname{Ext}_{R}^{i}(M, N)\right)_{\mu} \leq T^{i}\left(\begin{array}{c}
\mu-e_{i}+n-1 \\
n-1
\end{array}\right)
$$

(4) for any $j$ and any $\mu \geq e_{i}+j$,

$$
\operatorname{dim}_{k}\left(\operatorname{Tor}_{j}^{R}\left(\operatorname{Ext}_{R}^{i}(M, N), k\right)\right)_{\mu} \leq T^{i}\left(\begin{array}{c}
n \\
j
\end{array}\right)\left(\begin{array}{c}
\mu-e_{i}-j+n-1 \\
n-1
\end{array}\right) .
$$

Proof. For (1), see [CD 3.3]. We now prove (2), for which we may, and will, assume that $\operatorname{indeg}(M)=\operatorname{indeg}(N)=0$. Let $F_{\bullet}^{M}\left(\right.$ resp. $\left.F_{\bullet}^{N}\right)$ be a minimal free $R$-resolution of $M($ resp. $N)$ and set $C^{\bullet}:=\operatorname{Homgr}_{R}\left(F_{\bullet}^{M}, F_{\bullet}^{N}\right)$. Then $\operatorname{Ext}_{R}^{i}(M, N) \simeq H^{i}\left(C^{\bullet}\right)$. One has

$$
\begin{aligned}
& f^{i}:=\operatorname{indeg}\left(C^{i}\right)=\min _{p-q=i}\left\{f_{q}^{N}-b_{p}^{M}\right\} \geq-i-\operatorname{reg}(M), \\
& b^{i}:=\operatorname{reg}\left(C^{i}\right)=\max _{p-q=i}\left\{b_{q}^{N}-f_{p}^{M}\right\} \leq-i+\operatorname{reg}(N) .
\end{aligned}
$$

Set $K:=\operatorname{reg}(M)+\operatorname{reg}(N)+1$ and $\epsilon^{i}:=f^{i}+i+\operatorname{reg}(M) \geq 0$. By Corollary 2.3, it follows that

$$
\begin{aligned}
\operatorname{reg}\left(\operatorname{Ext}_{R}^{i}(M, N)\right) \leq & \max \left\{b^{i}, b^{i+1},\left[T^{i+1}\left(b^{i}-f^{i+1}\right)\right]^{2^{n-2}}+f^{i+1}+2,\right. \\
& {\left.\left[T^{i}\left(b^{i-1}-f^{i}\right)\right]^{2^{n-2}}+f^{i}\right\} } \\
\leq & \max \left\{\operatorname{reg}(N)-i,\left[T^{i+1}\left(K-\epsilon^{i+1}\right)\right]^{2^{n-2}}-i+1+\epsilon^{i+1},\right. \\
& \left.\quad\left[T^{i}\left(K-\epsilon^{i}\right)\right]^{2^{n-2}}-i+\epsilon^{i}\right\} \\
\leq & \max \left\{K T^{i}, K T^{i+1}\right\}^{2^{n-2}}+1-i .
\end{aligned}
$$

Finally (3) and (4) follow from the estimates in Lemma 2.2 (3) and (4).

Let $\mu(P)$ denote the minimal number of generators of a module $P$. The following lemma, in the spirit of some results above, can be used together with estimates on the regularities of $M$ and $N$ (see [CFN]) to bound the regularity of $\operatorname{Ext}_{R}^{i}(M, N)$ in terms of presentations of $M$ and $N$.

Lemma 2.5. For any $i$,

$$
\operatorname{dim}_{k} \operatorname{Tor}_{i}^{R}(M, k) \leq \mu(M)\left(\begin{array}{c}
n \\
i
\end{array}\right)\left(\begin{array}{c}
\operatorname{reg}(M)-\operatorname{indeg}(M)+n \\
n
\end{array}\right) .
$$


Proof. We may assume that $\operatorname{indeg}(M) \geq 0$. Then one has $\operatorname{Tor}_{i}^{S}(M, k) \simeq H_{i}(x ; M)$. In particular, $\operatorname{dim}_{k}\left(\operatorname{Tor}_{i}^{S}(M, k)\right)_{\mu} \leq\left(\begin{array}{c}n \\ i\end{array}\right) \operatorname{dim}_{k}\left(M_{\mu-i}\right) \leq\left(\begin{array}{c}n \\ i\end{array}\right) \mu(M)\left(\begin{array}{c}\mu-i+n-1 \\ n-1\end{array}\right)$. It follows that

$$
\operatorname{dim}_{k} \operatorname{Tor}_{i}^{R}(M, k) \leq\left(\begin{array}{c}
n \\
i
\end{array}\right) \mu(M) \sum_{\mu=i}^{\operatorname{reg}(M)+i}\left(\begin{array}{c}
\mu-i+n-1 \\
n-1
\end{array}\right),
$$

from which the claimed inequality follows.

\section{Refined estimate For the Regularity OF THE MODULES $\operatorname{Ext}_{R}^{i}(M, R)$}

Recall that $R$ is a standard graded polynomial ring in $n$ variables over a field $k$, and let $\mathfrak{m}$ be the maximal graded ideal of $R$. Let $M$ be a finitely generated graded $R$-module. Set $\bar{M}:=M / H_{\mathfrak{m}}^{0}(M)$ and $\Gamma M:=D_{\mathfrak{m}}(M) \simeq \bigoplus_{\mu} H^{0}\left(\mathbf{P}^{n-1}, \tilde{M}(\mu)\right)$.

We first make some remarks on the truncation of $M$.

Let $t$ be an integer and set $M^{\prime}:=M_{\geq t}$. For example, if $M=R / I$ and $t \geq 0$, then $M^{\prime}:=\mathfrak{m}^{t} /\left(I \cap \mathfrak{m}^{t}\right)$.

One has $\operatorname{Ext}_{R}^{i}\left(M^{\prime}, R\right)=\operatorname{Ext}_{R}^{i}(M, R)$ for $i<n-1$, and $\Gamma M=\Gamma M^{\prime}$. We also have $H_{\mathfrak{m}}^{0}\left(M^{\prime}\right)=H_{\mathfrak{m}}^{0}(M)_{\geq t}$, and the commutative diagram

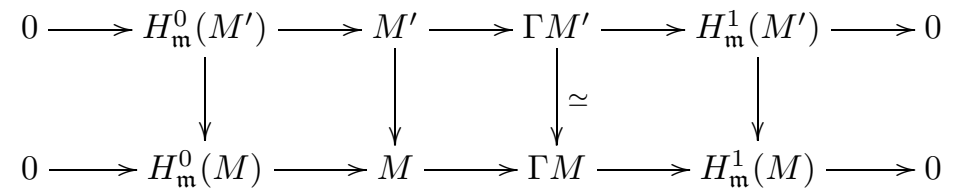

shows that $H_{\mathfrak{m}}^{1}\left(M^{\prime}\right)_{\geq t}=H_{\mathfrak{m}}^{1}(M)_{\geq t}$ and $H_{\mathfrak{m}}^{1}\left(M^{\prime}\right)_{\mu}=(\Gamma M)_{\mu}$ for $\mu<t$.

It follows that $\operatorname{reg}\left(M^{\prime}\right)=\max \{t, \operatorname{reg}(M)\}$. For a graded $R$-module $N$, set $N_{<t}:=$ $N /\left(N_{\geq t}\right)$. One has an exact sequence

$$
0 \rightarrow H_{\mathfrak{m}}^{0}(M)_{<t} \rightarrow M_{<t} \rightarrow H_{\mathfrak{m}}^{1}\left(M^{\prime}\right) \rightarrow H_{\mathfrak{m}}^{1}(M) \rightarrow 0,
$$

which gives by duality an exact sequence

$$
0 \rightarrow \operatorname{Ext}_{R}^{n-1}(M, R[-n]) \rightarrow \operatorname{Ext}_{R}^{n-1}\left(M^{\prime}, R[-n]\right) \rightarrow{ }^{*} \operatorname{Hom}_{R}\left(M / H_{\mathfrak{m}}^{0}(M), k\right)_{>-t} \rightarrow 0
$$

that in turn induces the exact sequence

$$
\begin{gathered}
0 \rightarrow H_{\mathfrak{m}}^{0}\left(\operatorname{Ext}_{R}^{n-1}(M, R)\right) \rightarrow H_{\mathfrak{m}}^{0}\left(\operatorname{Ext}_{R}^{n-1}\left(M^{\prime}, R\right)\right) \rightarrow{ }^{*} \operatorname{Hom}_{R}\left(M / H_{\mathfrak{m}}^{0}(M), k\right)[n]_{>-t-n} \\
(3.1) \quad \rightarrow H_{\mathfrak{m}}^{1}\left(\operatorname{Ext}_{R}^{n-1}(M, R)\right) \rightarrow H_{\mathfrak{m}}^{1}\left(\operatorname{Ext}_{R}^{n-1}\left(M^{\prime}, R\right) \rightarrow 0 .\right.
\end{gathered}
$$

Assume $t>\operatorname{indeg}(M)$ (i.e. $\left.M^{\prime} \neq M\right)$. Then ${ }^{*} \operatorname{Hom}_{R}\left(M / H_{\mathfrak{m}}^{0}(M), k\right)[n]_{>-t-n}$ is of finite length supported in degrees $\in\left[-t-n+1,-\operatorname{indeg}\left(M / H_{\mathfrak{m}}^{0}(M)\right)-n\right]$. It shows that

$$
\operatorname{reg}\left(\operatorname{Ext}_{R}^{n-1}(M, R)\right) \leq \max \left\{\operatorname{reg}\left(\operatorname{Ext}_{R}^{n-1}\left(M^{\prime}, R\right)\right),-\operatorname{indeg}\left(M / H_{\mathfrak{m}}^{0}(M)\right)-n\right\} .
$$

We gather direct consequences of the above facts in the following.

Remark 3.1. Let $M^{\prime}:=M_{\geq t}$. Then

(i) $\operatorname{reg}\left(M^{\prime}\right)=\max \{t, \operatorname{reg}(M)\}$,

(ii) $\operatorname{Ext}_{R}^{i}\left(M^{\prime}, R\right)=\operatorname{Ext}_{R}^{i}(M, R)$ for $i<n-1$,

(iii) $\operatorname{reg}\left(\operatorname{Ext}_{R}^{n-1}(M, R)\right) \leq \max \left\{\operatorname{reg}\left(\operatorname{Ext}_{R}^{n-1}\left(M^{\prime}, R\right)\right),-\operatorname{indeg}(M)-n\right\}$,

(iv) $\operatorname{Ext}_{R}^{n}\left(M^{\prime}, R\right)=\operatorname{Ext}_{R}^{n}(M, R)_{\leq-n-t}$, 
$(\mathrm{v}) \operatorname{Ext}_{R}^{n}(M, R)$ is a module of finite length whose intitial degree is - $\operatorname{end}\left(H_{\mathfrak{m}}^{0}(M)\right)$ $-n \geq-\operatorname{reg}(M)-n$ and whose regularity is $\operatorname{indeg}\left(H_{\mathfrak{m}}^{0}(M)\right)-n \leq-\operatorname{indeg}(M)-n$.

Furthermore,

$\mu\left(M^{\prime}\right)=\operatorname{dim}_{k}\left(\operatorname{Tor}_{0}^{R}\left(M^{\prime}, k\right)\right)=\operatorname{dim}_{k}\left(\operatorname{Tor}_{0}^{R}\left(M^{\prime}, k\right)\right)_{r}=H_{M}(r) \leq \mu(M)\left(\begin{array}{c}r+n-1 \\ n-1\end{array}\right)$.

Remark 3.2. Let $M$ be a finitely generated graded $R$-module of dimension $d$. To estimate regularity, we may assume $k$ is infinite. In this case, let $S$ be a polynomial ring in $d$ variables over $k$ inside $R$ such that $M$ is finite over $S$. We may assume that $S=k\left[X_{1}, \ldots, X_{d}\right]$. One has a graded isomorphism of $S$-modules

$$
\operatorname{Ext}_{R}^{i}(M, R) \simeq \operatorname{Ext}_{S}^{i-n+d}(M, S)[n-d]
$$

It follows that $\operatorname{Ext}_{R}^{i}(M, R)=0$ for $i<n-d$ and

$$
\operatorname{reg}\left(\operatorname{Ext}_{R}^{i}(M, R)\right)=\operatorname{reg}\left(\operatorname{Ext}_{S}^{i-n+d}(M, S)\right)-(n-d), \quad \forall i .
$$

Notice that this last equality can be written as

$$
\operatorname{reg}\left(\operatorname{Ext}_{R}^{i}(M, R)\right)+i=\operatorname{reg}\left(\operatorname{Ext}_{S}^{i-n+d}(M, S)\right)+(i-n+d), \quad \forall i .
$$

We will need the following formula for the Betti numbers of a module with a linear resolution, which may be of use for other applications.

Proposition 3.3. Let $M$ be a finitely generated graded $R$-module with $\operatorname{reg}(M)=$ $\operatorname{indeg}(M)=: r$ and $H_{\mathfrak{m}}^{0}(M)=0$. Set $M^{\prime}:=(M / l M)_{\geq r+1}$ for a linear non-zero divisor $l$. Then

$$
\operatorname{dim}_{k} \operatorname{Tor}_{i}^{R}(M, k)=\left(\begin{array}{c}
n-1 \\
i
\end{array}\right) P_{M}(r)-\operatorname{dim}_{k} \operatorname{Tor}_{i-1}^{R / l R}\left(M^{\prime}, k\right)
$$

Proof. Recall that if $N$ is a module with indeg $(N)=\operatorname{reg}(N)=s$, then $\operatorname{Tor}_{i}^{R}(N, k)$ is concentrated in degree $s+i$ for all $i$. Notice that $\operatorname{reg}\left(M_{\geq r+1}\right)=\operatorname{indeg}\left(M_{\geq r+1}\right)=$ $r+1$, that $M^{\prime}=0$ if $\operatorname{dim} M=1$, and that $\operatorname{reg}\left(M^{\prime}\right)=\operatorname{indeg}\left(M^{\prime}\right)=r+1$ if $\operatorname{dim} M \geq 2$. We induct on $i$. The case $i=-1$ is trivially satisfied $(i=0$ is also clear). The exact sequences

$$
0 \rightarrow M(-1) \rightarrow M_{\geq r+1} \rightarrow M^{\prime} \rightarrow 0
$$

and

$$
0 \rightarrow M_{\geq r+1} \rightarrow M \rightarrow M_{r} \rightarrow 0
$$

induce exact sequences

$$
0 \rightarrow \operatorname{Tor}_{i}^{R}(M, k) \rightarrow \operatorname{Tor}_{i}^{R}\left(M_{\geq r+1}, k\right) \rightarrow \operatorname{Tor}_{i}^{R}\left(M^{\prime}, k\right) \rightarrow 0
$$

and

$$
0 \rightarrow \operatorname{Tor}_{i+1}^{R}(M, k) \rightarrow \operatorname{Tor}_{i+1}^{R}\left(M_{r}, k\right) \rightarrow \operatorname{Tor}_{i}^{R}\left(M_{\geq r+1}, k\right) \rightarrow 0
$$


which shows that

$$
\begin{aligned}
\operatorname{dim}_{k} \operatorname{Tor}_{i+1}^{R}(M, k)= & \operatorname{dim}_{k} \operatorname{Tor}_{i+1}^{R}\left(M_{r}, k\right)-\operatorname{dim}_{k} \operatorname{Tor}_{i}^{R}(M, k)-\operatorname{dim}_{k} \operatorname{Tor}_{i}^{R}\left(M^{\prime}, k\right) \\
= & \left(\begin{array}{c}
n \\
i+1
\end{array}\right) P(r)-\operatorname{dim}_{k} \operatorname{Tor}_{i}^{R}(M, k)-\operatorname{dim}_{k} \operatorname{Tor}_{i}^{R / l R}\left(M^{\prime}, k\right) \\
& \quad-\operatorname{dim}_{k} \operatorname{Tor}_{i-1}^{R / l R}\left(M^{\prime}, k\right) \\
& =\left(\begin{array}{c}
n \\
i+1
\end{array}\right) P(r)-\left(\begin{array}{c}
n-1 \\
i
\end{array}\right) P_{M}(r)-\operatorname{dim}_{k} \operatorname{Tor}_{i}^{R / l R}\left(M^{\prime}, k\right) \\
& =\left(\begin{array}{c}
n-1 \\
i+1
\end{array}\right) P_{M}(r)-\operatorname{dim}_{k} \operatorname{Tor}_{i}^{R / l R}\left(M^{\prime}, k\right)
\end{aligned}
$$

by induction on $i$.

For a polynomial $P$, set $\Delta P(t):=P(t)-P(t-1)$ and $\Delta^{i} P:=\Delta\left(\Delta^{i-1} P\right)$.

Corollary 3.4. Let $M$ be a finitely generated graded $R$-module of dimension d with $\operatorname{reg}(M)=\operatorname{indeg}(M)=: r$ and $H_{\mathfrak{m}}^{0}(M)=0$. Then

$$
\operatorname{dim}_{k} \operatorname{Tor}_{i}^{R}(M, k)=\sum_{\ell=0}^{\min \{i, d-1\}}(-1)^{\ell}\left(\begin{array}{c}
n-\ell-1 \\
i-\ell
\end{array}\right) \Delta^{\ell} P_{M}(r+\ell) .
$$

Proof. Notice that $M^{\prime}$ has positive depth, because $M / l M$ has regularity $r$. As $M^{\prime}$ has regularity $r+1$, Hilbert polynomial $\Delta^{1} P_{M}$, and $R / l R$ is isomorphic to a polynomial ring in $n-1$ variables, the claim follows by induction.

Theorem 3.5. Set $d:=\operatorname{dim} M$ and $\bar{r}:=\operatorname{reg}\left(M / H_{\mathfrak{m}}^{0}(M)\right)$.

(1) If $d<2$, then $\operatorname{reg}\left(\operatorname{Ext}_{R}^{i}(M, R) \leq-\operatorname{indeg}(M)-i\right.$ for any $i$.

(2) If $d \geq 2$, then

(a) $\operatorname{reg}\left(\operatorname{Ext}_{R}^{n}(M, R)+n \leq-\operatorname{indeg}(M)\right.$,

(b) $\operatorname{reg}\left(\operatorname{Ext}_{R}^{n-1}(M, R)\right)+(n-1) \leq \max \left\{P_{M}(\bar{r})-\Delta^{1} P_{M}(\bar{r})-\bar{r}\right.$, $-\operatorname{indeg}(M)-1\}$,

(c) for $i>1$

$$
\operatorname{reg}\left(\operatorname{Ext}_{R}^{n-i}(M, R)\right)+(n-i) \leq\left[C_{d, d-i} P_{M}(\bar{r})\right]^{2^{d-2}}-\bar{r}+1,
$$

with $C_{d, j}:=\max \left\{\left(\begin{array}{c}d-1 \\ j\end{array}\right),\left(\begin{array}{c}d-1 \\ j+1\end{array}\right)\right\}$.

Proof. (1) Recall that $\operatorname{Ext}_{R}^{i}(M, R)=0$ for $i<n-d$. By Remark 3.1 (v), it remains to check that the inequality holds when $d=1$ and $i=n-1$. When $d=1$, $\operatorname{Ext}_{R}^{n-1}(M, R) \simeq \operatorname{Ext}_{R}^{n-1}\left(M / H_{\mathfrak{m}}^{0}(M), R\right)$, which shows that $\operatorname{reg}\left(\operatorname{Ext}_{R}^{n-1}(M, R)\right)=$ - $\operatorname{indeg}\left(M / H_{\mathfrak{m}}^{0}(M)\right)-(n-1) \leq-\operatorname{indeg}(M)-(n-1)$, because $M / H_{\mathfrak{m}}^{0}(M)$ is Cohen-Macaulay of dimension 1.

(2)(a) was proved in Remark3.1(v). For (2)(b) and (2)(c), by Remark3.1(i)-(iii) and Remark 3.2 we are reduced to showing this estimate for $M$ with indeg $(M)=$ $\operatorname{reg}(M)$ and $d=n$. Applying Corollary 2.3 to the $R$-dual of a minimal free $R$ resolution of $M$, we deduce that, setting $T_{i}:=\operatorname{dim}_{k} \operatorname{Tor}_{i}^{R}(M, k)$, one has

$$
\operatorname{reg}\left(\operatorname{Ext}_{R}^{i}(M, R)\right) \leq \max \left\{T_{i}^{2^{n-2}}, T_{i+1}^{2^{n-2}}+1\right\}-\bar{r}-i .
$$

Hence the conclusion follows from Proposition 3.3 


\section{Hilbert function AND Hilbert COEFficients}

In this section we will estimate graded components of the Hilbert function of $\operatorname{Ext}_{R}^{i}(M, R)$. Based on such an estimation we will give bounds for the Hilbert coefficients in terms of the Castelnuovo-Mumford regularity of $M$.

Lemma 4.1. Let $\bar{M}:=M / H_{\mathfrak{m}}^{0}(M)$ and $\bar{r}=\operatorname{reg}(\bar{M})$. Then

(i) $P_{M}(t)=H_{\bar{M}}(t)$ for all $t \geq \bar{r}$ and $P_{M}(t)$ is increasing for all $t \geq \bar{r}-1$.

(ii) If $\operatorname{dim} M \geq 1$, then $H_{\bar{M}}(\bar{r}) \geq \operatorname{deg}(M)$.

Proof. (i) By the Grothendieck-Serre formula,

$$
H_{\bar{M}}(t)-P_{\bar{M}}(t)=\sum_{i=1}^{d}(-1)^{i} \ell\left(H_{\mathfrak{m}}^{i}(\bar{M})_{t}\right) .
$$

This implies that $P_{M}(t)=P_{\bar{M}}(t)=H_{\bar{M}}(t)$ for all $t \geq \bar{r}$. Since $H_{\bar{M}}(t)$ is an increasing function, $P_{\bar{M}}(t)$ is also increasing for all $t \geq \bar{r}$. If $d \leq 1$, then $P_{M}(t)$ is a constant. Let $d \geq 2$ and $l$ be a generic linear form. Then

$$
P_{M}(\bar{r})-P_{M}(\bar{r}-1)=P_{M / l M}(\bar{r})=H_{\overline{M / l M}}(\bar{r}) \geq 0 .
$$

(ii) If $\operatorname{dim} M=1$, then by (i) $H_{\bar{M}}(\bar{r})=P_{M}(\bar{M})=\operatorname{deg}(M)$. If $d=\operatorname{dim} M \geq 1$, let $l_{1}, \ldots, l_{d-1}$ be generic linear forms. Since $\operatorname{reg}\left(\bar{M} /\left(l_{1}, \ldots, l_{d-1}\right) \bar{M}\right) \leq \bar{r}$, the above remark implies that

$$
H_{\bar{M}}(\bar{r}) \geq H_{\bar{M} /\left(l_{1}, \ldots, l_{d-1}\right) \bar{M}}(\bar{r})=P_{\overline{\bar{M} /\left(l_{1}, \ldots, l_{d-1}\right) \bar{M}}}(\bar{r})=\operatorname{deg}(M)
$$

Theorem 4.2. Let $M$ be a finitely generated graded $R$-module of dimension $d \geq$ 1. Let $l_{1}, \ldots, l_{d}$ be a filter regular sequence of linear forms on $M$ and $M_{j}:=$ $M /\left(l_{1}, \ldots l_{j}\right) M$. Set $\overline{M_{j}}:=M_{j} / H_{\mathfrak{m}}^{0}\left(M_{j}\right)$ and $\bar{r}_{j}:=\operatorname{reg}\left(\overline{M_{j}}\right)$. Then for $i>0$, $\operatorname{indeg}\left(\operatorname{Ext}_{R}^{n-i}(M, R)\right) \geq-\bar{r}_{i-1}-n+1$ and

$$
\operatorname{dim}_{k} \operatorname{Ext}_{R}^{n-i}(M, R)_{\mu} \leq\left(\begin{array}{c}
\mu+\bar{r}_{i-1}+n-1 \\
i-1
\end{array}\right) \Delta^{i-1} P_{M}\left(\bar{r}_{i}-1\right) .
$$

Proof. First notice that $\operatorname{Ext}_{R}^{i}\left(M_{j}, R\right)=\operatorname{Ext}_{R}^{i}\left(\overline{M_{j}}, R\right)$ for $i \neq n$.

Set $N_{j+1}:=\overline{M_{j}} / l_{j+1} \overline{M_{j}}$. One has $\operatorname{reg}\left(N_{j+1}\right)=\operatorname{reg}\left(\overline{M_{j}}\right)=\bar{r}_{j}$ and

$$
N_{j+1} \cong M_{j} /\left(l_{j+1} M_{j}+H_{\mathfrak{m}}^{0}\left(M_{j}\right)\right) \cong M_{j+1} /\left(\left(l_{j+1} M_{j}+H_{\mathfrak{m}}^{0}\left(M_{j}\right)\right) / l_{j+1} M_{j}\right) .
$$

Noticing that the module $U:=\left(l_{j+1} M_{j}+H_{\mathfrak{m}}^{0}\left(M_{j}\right)\right) / l_{j+1} M_{j} \cong H_{\mathfrak{m}}^{0}\left(M_{j}\right) /\left(H_{\mathfrak{m}}^{0}\left(M_{j}\right) \cap\right.$ $\left.l_{j+1} M_{j}\right)$ is of finite length, we get that $U$ is a submodule of $H_{\mathfrak{m}}^{0}\left(M_{j+1}\right)$ and that $H_{\mathfrak{m}}^{0}\left(M_{j+1}\right) / U \cong H_{\mathfrak{m}}^{0}\left(N_{j+1}\right)$. Hence

$$
\overline{M_{j+1}} \cong\left(M_{j+1} / U\right) /\left(H_{\mathfrak{m}}^{0}\left(M_{j+1}\right) / U\right) \cong N_{j+1} / H_{\mathfrak{m}}^{0}\left(N_{j+1}\right)
$$

which also shows that $\bar{r}_{j+1} \leq \bar{r}_{j}$.

Now we show by induction on $i \geq 1$ that

$$
\operatorname{indeg}\left(\operatorname{Ext}_{R}^{n-i}\left(M_{j}, R\right)\right) \geq \bar{r}_{j+i-1}-n+i
$$

and that

$$
\operatorname{dim}_{k} \operatorname{Ext}_{R}^{n-i}\left(M_{j}, R\right)_{\mu} \leq\left(\begin{array}{c}
\mu+\bar{r}_{j+i-1}+n-1 \\
i-1
\end{array}\right) P_{M_{j+i-1}}\left(\bar{r}_{j+i}-1\right)
$$

for all $j \geq 0$. 
Let $i=1$. In this case, by Lemma 4.1(i), $H_{\overline{M_{j}}}(\nu)=P_{\overline{M_{j}}}(\nu)=P_{M_{j}}(\nu)$ for $\nu \geq \operatorname{reg}\left(\overline{M_{j}}\right)$. Recall that $P_{M_{j+1}}(\nu)=P_{M_{j}}(\nu)-P_{M_{j}}(\nu-1)$ for any $\nu$. The exact sequence

$$
0 \rightarrow \overline{M_{j}}(-1) \rightarrow \overline{M_{j}} \rightarrow N_{j+1} \rightarrow 0
$$

induces, for $i<n$, an exact sequence

$$
\cdots \rightarrow \operatorname{Ext}_{R}^{i}\left(M_{j}, R\right) \rightarrow \operatorname{Ext}_{R}^{i}\left(M_{j}, R\right)(1) \rightarrow \operatorname{Ext}_{R}^{i+1}\left(N_{j+1}, R\right),
$$

which shows that, for $i<n-1$,

$$
\operatorname{dim}_{k} \operatorname{Ext}_{R}^{i}\left(M_{j}, R\right)_{\mu} \leq \sum_{\nu<\mu} \operatorname{dim}_{k} \operatorname{Ext}_{R}^{i+1}\left(N_{j+1}, R\right)_{\nu}
$$

and

$$
\begin{aligned}
\operatorname{dim}_{k} \operatorname{Ext}_{R}^{n-1}\left(M_{j}, R\right)_{\mu} & \leq \sum_{\nu<\mu} \operatorname{dim}_{k} \operatorname{Ext}_{R}^{n}\left(N_{j+1}, R\right)_{\nu} \\
& =\sum_{\nu<\mu} \operatorname{dim}_{k} H_{\mathfrak{m}}^{0}\left(N_{j+1}\right)_{-\nu-n} \\
& =\sum_{\nu=-n-\mu+1}^{\operatorname{end}\left(H_{\mathfrak{m}}^{0}\left(N_{j+1}\right)\right)} \operatorname{dim}_{k} H_{\mathfrak{m}}^{0}\left(N_{j+1}\right)_{\nu} \\
& \leq \sum_{\nu \leq \bar{r}_{j}}\left(H_{N_{j+1}}(\nu)-H_{\overline{M_{j+1}}}(\nu)\right) \\
& =H_{\bar{M}_{j}}\left(\bar{r}_{j}\right)-\sum_{\nu \leq \bar{r}_{j}} H_{\overline{M_{j+1}}}(\nu) \\
& \left.\leq P_{M_{j}}\left(\bar{r}_{j}\right)-\sum_{\bar{r}_{j+1} \leq \nu \leq \bar{r}_{j}} P_{M_{j+1}}(\nu) \quad \text { (by Lemma 4.1 }(\mathrm{i})\right) \\
& =P_{M_{j}}\left(\bar{r}_{j}\right)-\sum_{\bar{r}_{j+1} \leq \nu \leq \bar{r}_{j}}\left(P_{M_{j}}(\nu)-P_{M_{j}}(\nu-1)\right) \\
& =P_{M_{j}}\left(\bar{r}_{j+1}-1\right) .
\end{aligned}
$$

By (1) in Theorem 2.4, indeg $\left(\operatorname{Ext}_{R}^{n-1}\left(M_{j}, R\right)\right) \geq-\bar{r}_{j}-n+1$. This means that $\operatorname{Ext}_{R}^{n-1}\left(M_{j}, R\right)_{\mu}=0$ for all $\mu \leq-\bar{r}_{j}-n$. For $\mu \geq-\bar{r}_{j}-n+1,\left(\begin{array}{c}\mu+\bar{r}_{j}+n-1 \\ 0\end{array}\right)=1$. Hence (4.6) implies the claim for $i=1$ and all $j$.

Let $i \geq 2$. Notice that

$$
\operatorname{Ext}_{R}^{n-i+1}\left(N_{j+1}, R\right) \simeq \operatorname{Ext}_{R}^{n-i+1}\left(\overline{M_{j+1}}, R\right) \simeq \operatorname{Ext}_{R}^{n-i+1}\left(M_{j+1}, R\right) .
$$

Furthermore, $M_{j+1}$ is of dimension $d-j-1$ and $l_{j+2}, \ldots, l_{d}$ is a filter regular sequence on $M_{j+1}$. One has

$$
\begin{aligned}
\operatorname{dim}_{k} \operatorname{Ext}_{R}^{n-i}\left(M_{j}, R\right)_{\mu} & \left.\leq \sum_{\nu<\mu} \operatorname{dim}_{k} \operatorname{Ext}_{R}^{n-i+1}\left(N_{j+1}, R\right)_{\nu} \quad \text { (by (4.4) and (4.2) }\right) \\
& =\sum_{\nu<\mu} \operatorname{dim}_{k} \operatorname{Ext}_{R}^{n-(i-1)}\left(M_{j+1}, R\right)_{\nu} .
\end{aligned}
$$


By induction hypothesis, $\operatorname{indeg}\left(\operatorname{Ext}_{R}^{n-(i-1)}\left(M_{j+1}, R\right)\right) \geq-\bar{r}_{(j+1)+(i-2)}-n+i-1=$ $-\bar{r}_{j+i-1}-n+i-1$, and therefore

$$
\begin{aligned}
\operatorname{dim}_{k} \operatorname{Ext}_{R}^{n-i}\left(M_{j}, R\right)_{\mu} & \leq \sum_{\nu=-\bar{r}_{j+i-1}-n+i-1}^{\mu-1} \operatorname{dim}_{k} \operatorname{Ext}_{R}^{n-(i-1)}\left(M_{j+1}, R\right)_{\nu} \\
& \leq \sum_{\nu=-\bar{r}_{j+i-1}-n+i-1}^{\mu-1}\left(\begin{array}{c}
\nu+\bar{r}_{j+i-1}+n-1 \\
i-2
\end{array}\right) \Delta^{i-2} P_{M_{j+1}}\left(\bar{r}_{j+i}-1\right) \\
& =\sum_{\nu=i-2}^{\mu+\bar{r}_{j+i-1}+n-2}\left(\begin{array}{c}
\nu \\
i-2
\end{array}\right) P_{M_{j+i-1}}\left(\bar{r}_{j+i}-1\right) \\
& =\left(\begin{array}{c}
\mu+\bar{r}_{j+i-1}+n-1 \\
i-1
\end{array}\right) P_{M_{j+i-1}}\left(\bar{r}_{j+i}-1\right) .
\end{aligned}
$$

Finally, using (4.3) we get epimorphisms

$$
\operatorname{Ext}_{R}^{n-i}\left(M_{j}, R\right)_{\mu} \rightarrow \operatorname{Ext}_{R}^{n-i}\left(M_{j}, R\right)_{\mu+1} \rightarrow 0
$$

for all $\mu<\bar{r}_{j+i-1}-n+i-1$. Since $\operatorname{Ext}_{R}^{n-i}\left(M_{j}, R\right)_{\mu}=0$ for $\mu \ll 0$, this yields $\operatorname{Ext}_{R}^{n-i}\left(M_{j}, R\right)_{\mu}=0$ for all $\mu \leq \bar{r}_{j+i-1}-n+i-1$. Hence indeg $\left(\operatorname{Ext}_{R}^{n-i}\left(M_{j}, R\right)\right) \geq$ $\bar{r}_{j+i-1}-n+i$, as required.

In particular,

$$
\begin{aligned}
& \operatorname{dim}_{k} \operatorname{Ext}_{R}^{n-d}(M, R)_{\mu} \leq\left(\begin{array}{c}
\mu+\bar{r}_{d-1}+n-1 \\
d-1
\end{array}\right) \operatorname{deg} M \\
& \operatorname{dim}_{k} \operatorname{Ext}_{R}^{n-d+1}(M, R)_{\mu} \leq\left(\begin{array}{c}
\mu+\bar{r}_{d-2}+n-1 \\
d-2
\end{array}\right) P_{M_{d-2}}\left(\bar{r}_{d-1}-1\right)
\end{aligned}
$$

and the numbers $\bar{r}_{d-1} \leq r_{d}$ and $\bar{r}_{d-2} \leq r_{d-1}$ can be quite sharply estimated from the degrees of generators and relations of $M$ by [CFN, 2.1].

For later use we also need a bound in terms of the Hilbert function.

Corollary 4.3. Keep the notation of Theorem 4.2. Then for $i>0$,

$\operatorname{dim}_{k} \operatorname{Ext}_{R}^{n-i}(M, R)_{\mu} \leq\left(\begin{array}{c}\mu+\bar{r}_{i-1}+n-1 \\ i-1\end{array}\right) H_{\overline{M_{i-1}}}\left(\bar{r}_{i-1}\right) \leq\left(\begin{array}{c}\mu+\bar{r}+n-1 \\ i-1\end{array}\right) H_{\bar{M}}(\bar{r})$.

Proof. The second inequality follows from the first one by using the fact $\bar{r}_{i-1} \leq \bar{r}$.

To prove the first inequality, first note from (4.5) that $\operatorname{dim}_{k} \operatorname{Ext}_{R}^{n-1}\left(M_{j}, R\right)_{\mu} \leq$ $P_{M_{j}}\left(\bar{r}_{j}\right)$. Using this inequality instead of (4.6) in the last induction step of the above theorem we get

$$
\operatorname{dim}_{k} \operatorname{Ext}_{R}^{n-i}(M, R)_{\mu} \leq\left(\begin{array}{c}
\mu+\bar{r}_{i-1}+n-1 \\
i-1
\end{array}\right) \Delta^{i-1} P_{M}\left(\bar{r}_{i-1}\right)
$$

Further, note that $\Delta^{i-1} P_{M}(t)=P_{M_{i-1}}(t)$. Since this polynomial is increasing for all $t \geq \bar{r}_{i-1}$ and $P_{M_{i-1}}\left(\bar{r}_{i-1}\right)=H_{\overline{M_{i-1}}}\left(\bar{r}_{i-1}\right)$ (by Lemma 4.1(i)), the claim follows from the above inequality.

Lemma 4.4. Assume that $M$ is a finitely generated graded $R$-module of dimension $d \geq 1$ and indeg $M=0$. Let $l_{1}, \ldots, l_{d}$ be a filter regular sequence of linear forms on $M$ and $B=\operatorname{dim}_{k}\left(M /\left(l_{1}, \ldots, l_{d}\right) M\right)$. Then

(i) $H_{M}(\mu) \leq B\left(\begin{array}{c}\mu+d-1 \\ d-1\end{array}\right)$,

(ii) $H_{M}(\mu) \leq \mu(M)\left(\begin{array}{c}\mu+n-1 \\ n-1\end{array}\right)$. 
Proof. (i) We do induction on $d$. Let $d=1$. From the exact sequence

$$
0 \rightarrow\left(0: l_{1}\right)_{\mu-1} \rightarrow M_{\mu-1} \rightarrow M_{\mu} \rightarrow\left(M / l_{1} M\right)_{\mu} \rightarrow 0
$$

and $M_{-1}=0$, we get

$$
\begin{aligned}
\operatorname{dim}_{k}\left(M_{\mu}\right) & \leq \operatorname{dim}_{k}\left(M_{\mu-1}\right)+\operatorname{dim}_{k}\left(\left(M / l_{1} M\right)_{\mu}\right) \\
& \leq \cdots \leq \sum_{j=0}^{\mu} \operatorname{dim}_{k}\left(\left(M / l_{1} M\right)_{j}\right) \leq \operatorname{dim}_{k}\left(M / l_{1} M\right)=B .
\end{aligned}
$$

Let $d \geq 2$. As above,

$$
\operatorname{dim}_{k}\left(M_{\mu}\right) \leq \sum_{j=0}^{\mu} \operatorname{dim}_{k}\left(\left(M / l_{d} M\right)_{j}\right) .
$$

An application of the induction hypothesis yields

$$
\operatorname{dim}_{k}\left(M_{\mu}\right) \leq \operatorname{dim}_{k}\left(M /\left(l_{1}, \ldots, l_{d}\right) M\right) \sum_{j=0}^{s}\left(\begin{array}{c}
j+d-2 \\
d-2
\end{array}\right)=B\left(\begin{array}{c}
\mu+d-1 \\
d-1
\end{array}\right) .
$$

(ii) This is clear if we present $M$ as a factor module of the free module $\bigoplus_{j=1}^{\mu(M)}$ $R\left(-a_{j}\right)$, where $a_{j} \geq 0$ is an integer for all $j$.

The following bounds do not depend on the Hilbert function of $M$. It is an extension of [ $\underline{\mathrm{H}}$, Theorem 3.4] to the case of modules. Note that our proof here is completely different from that in $[\mathrm{H}]$.

Theorem 4.5. Assume that $M$ is a finitely generated graded $R$-module of dimension $d \geq 1$ and indeg $M=0$. Let $l_{1}, \ldots, l_{d}$ be a filter regular sequence of linear forms on $M$, and set $M_{i}:=M /\left(l_{1}, \ldots, l_{i}\right) M, B:=\operatorname{dim}_{k}\left(M_{d}\right)$ and $\bar{r}_{i}=\operatorname{reg}\left(\overline{M_{i}}\right)$. Then for all $0<i \leq n$ we have

(i) $\operatorname{dim}_{k} \operatorname{Ext}_{R}^{n-i}(M, R)_{\mu} \leq B\left(\begin{array}{c}\bar{r}_{i-1}+d-i \\ d-i\end{array}\right)\left(\begin{array}{c}\mu+\bar{r}_{i-1}+n-1 \\ i-1\end{array}\right)$,

(ii) $\operatorname{dim}_{k} H_{\mathfrak{m}}^{i}(M)_{\mu} \leq B\left(\begin{array}{c}\bar{r}_{i-1}+d-i \\ d-i\end{array}\right)\left(\begin{array}{c}-\mu+\bar{r}_{i-1}-1 \\ i-1\end{array}\right)$.

Proof. The second statement follows from the first one and the graded isomorphism $\operatorname{Hom}\left(H_{\mathfrak{m}}^{i}(M), k\right) \cong \operatorname{Ext}_{R}^{n-i}(M, R)(-n)$. To prove the first statement, applying Lemma 4.4 to $\overline{M_{i-1}}$ we get

$$
H_{\overline{M_{i-1}}}\left(\bar{r}_{i-1}\right) \leq B\left(\begin{array}{c}
\bar{r}_{i-1}+d-i \\
d-i
\end{array}\right)
$$

The result then follows from Corollary 4.3 .

Write the Hilbert polynomial of $M$ in the form

$$
P_{M}(t)=e_{0}(M)\left(\begin{array}{c}
t+d-1 \\
d-1
\end{array}\right)-e_{1}(M)\left(\begin{array}{c}
t+d-2 \\
d-2
\end{array}\right)+\cdots+(-1)^{d-1} e_{d-1}(M) .
$$

Then $e_{0}(M), e_{1}(M), \ldots, e_{d-1}(M)$ are called Hilbert coefficients of $M$. Note that $e_{0}(M)=\operatorname{deg}(M)$. Applying the above estimates we can bound the Hilbert coefficients in terms of the Castelnuovo-Mumford regularity of $M$. The following result extends Theorem 4.1 and Theorem 4.6 in $[\mathrm{H}]$. Moreover, the bound here is also a little bit better. 
Theorem 4.6. Assume that $M$ is a finitely generated graded $R$-module of dimension $d \geq 1$ and $\operatorname{indeg} M=0$. Let $l_{1}, \ldots, l_{d}$ be a filter regular sequence of linear forms on $M$ and $B=\operatorname{dim}_{k}\left(M /\left(l_{1}, \ldots, l_{d}\right) M\right)$. Then for all $0 \leq i \leq d-1$ we have

$$
\left|e_{i}(M)\right| \leq B \cdot(\operatorname{reg}(\bar{M})+1)^{i} .
$$

Proof. As usual we set $\bar{r}=\operatorname{reg}(\bar{M})$. We do induction on $d$. Note that $0 \leq e_{0}(M) \leq$ $B$. Hence the inequality holds true for $i=0$. In particular the statement holds for $d=1$. Assume that the statement holds for all modules of dimension $d-1 \geq 1$. Let $M$ be a module of dimension $d$ and $M_{1}=M / l_{1} M$. Then $e_{i}(M)=e_{i}\left(M_{1}\right)$ for all $i \leq d-2$. Since $\operatorname{reg}\left(\overline{M_{1}}\right) \leq \operatorname{reg}(\bar{M})$ and $\operatorname{dim}_{k}\left(M_{1} /\left(l_{2}, \ldots, l_{d}\right) M_{1}\right)=B$, by the induction hypothesis it suffices to show the inequality

$$
\left|e_{d-1}(M)\right| \leq B(\bar{r}+1)^{d-1} .
$$

Note that we may assume $M=\bar{M}$, i.e. $H_{\mathfrak{m}}^{0}(M)=0$. From the Grothendieck-Serre formula (4.1) we get (setting $t=-1$ )

$$
(-1)^{d-1} e_{d-1}(M)=C_{d}-D_{d},
$$

where

$$
C_{d}=\operatorname{dim}_{k}\left(H_{\mathfrak{m}}^{1}(M)_{-1}\right)+\operatorname{dim}_{k}\left(H_{\mathfrak{m}}^{3}(M)_{-1}\right) \cdots
$$

and

$$
D_{d}=\operatorname{dim}_{k}\left(H_{\mathfrak{m}}^{2}(M)_{-1}\right)+\operatorname{dim}_{k}\left(H_{\mathfrak{m}}^{4}(M)_{-1}\right) \cdots .
$$

By Theorem 4.5(ii) we have

$$
C_{d} \leq B \sum_{1 \leq 2 j+1 \leq d}\left(\begin{array}{c}
\bar{r} \\
2 j
\end{array}\right)\left(\begin{array}{c}
\bar{r}+d-2 j-1 \\
d-2 j-1
\end{array}\right)=: B . \tilde{C}_{d} .
$$

We show by induction on $d$ that $\tilde{C}_{d} \leq(\bar{r}+1)^{d-1}$. We have $\tilde{C}_{2}=\bar{r}+1$ and $\tilde{C}_{3}=\bar{r}^{2}+\bar{r}+1<(\bar{r}+1)^{2}$. Let $d \geq 4$. Assume that

$$
\tilde{C}_{d-1} \leq(\bar{r}+1)^{d-2} .
$$

If $d$ is even, then $d-2 j-1 \geq 1$ and

$$
\frac{\bar{r}+d-2 j-1}{d-2 j-1}=\frac{\bar{r}+d-2 j-1}{d-2 j-1} \frac{\bar{r}+(d-1)-2 j-1}{(d-1)-2 j-1} \leq(\bar{r}+1) \frac{\bar{r}+(d-1)-2 j-1}{(d-1)-2 j-1} .
$$

Hence, by the induction hypothesis on $\tilde{C}_{d-1}$ we get

$$
\tilde{C}_{d} \leq(\bar{r}+1) \sum_{1 \leq 2 j+1 \leq d}\left(\begin{array}{c}
\bar{r} \\
2 j
\end{array}\right)\left(\begin{array}{c}
\bar{r}+(d-1)-2 j-1 \\
(d-1)-2 j-1
\end{array}\right)=(\bar{r}+1) \tilde{C}_{d-1} \leq(\bar{r}+1)^{d-1} .
$$

If $d$ is odd, say $d=2 \delta+1$, then for $j<\delta$ we have $d-2 j-1 \geq 2$ and (4.7)

$$
\frac{\bar{r}+d-2 j-1}{d-2 j-1}=\left(\frac{\bar{r}}{d-2 j-1}+1\right) \frac{\bar{r}+(d-1)-2 j-1}{(d-1)-2 j-1} \leq\left(\frac{\bar{r}}{2}+1\right) \frac{\bar{r}+(d-1)-2 j-1}{(d-1)-2 j-1} .
$$


Therefore

$$
\begin{aligned}
\tilde{C}_{d} & \leq\left(\frac{\bar{r}}{2}+1\right) \sum_{1 \leq 2 j+1 \leq d-1}\left(\begin{array}{c}
\bar{r} \\
2 j
\end{array}\right)\left(\begin{array}{c}
\bar{r}+(d-1)-2 j-1 \\
(d-1)-2 j-1
\end{array}\right)+\left(\begin{array}{c}
\bar{r} \\
d-1
\end{array}\right) \\
& <\left(\frac{\bar{r}}{2}+1\right) \tilde{C}_{d-1}+\frac{(\bar{r}+1)^{d-2} \bar{r}}{2} \\
& \leq\left(\frac{\bar{r}}{2}+1\right)(\bar{r}+1)^{d-2}+(\bar{r}+1)^{d-2} \frac{\bar{r}}{2} \\
& =(\bar{r}+1)^{d-1}
\end{aligned}
$$

Thus we have proved $\tilde{C}_{d} \leq(\bar{r}+1)^{d-1}$, and so $C_{d} \leq B(\bar{r}+1)^{d-1}$. Similarly, $D_{d} \leq B(\bar{r}+1)^{d-1}$. Hence

$$
\left|e_{d-1}(M)\right| \leq \max \left\{C_{d}, D_{d}\right\} \leq B(\bar{r}+1)^{d-1},
$$

as required.

Remark 4.7. (i) If $M$ is a Cohen-Macaulay module, then $B=\operatorname{deg}(M)$. In this case the bound of Theorem 4.6 is related to the bound given in HHy1, Lemma 11]. If $M=R / I$, where $I$ is a homogeneous ideal generated by forms of degrees at most $\Delta$, then

$$
B \leq \max \left\{\Delta^{n-d}, \operatorname{adeg}(M)^{n-d}\right\},
$$

where $\operatorname{adeg}(M)=\sum_{i} \operatorname{deg}\left(\operatorname{Ext}_{R}^{i}\left(\operatorname{Ext}_{R}^{i}(M, R), R\right)\right)$ is the so-called arithmetic degree of $M$; see the proof of [H, Theorem3.4].

(ii) Considering $M /\left(l_{1}, \ldots, l_{d}\right) M$ as a module over $R /\left(l_{1}, \ldots, l_{d}\right) R$, by Lemma 4.4(ii) we have

$$
B \leq \mu(M)\left(\begin{array}{c}
\bar{r}+n-d \\
n-d
\end{array}\right) .
$$

(iii) Example 4.9 in $[\mathrm{H}]$ shows that the bound on Hilbert coefficients given in the above theorem is rather good.

\section{A BOUnd FOR THE HOMOLOGICAL DEGREe}

The homological degree of a finite graded $R$-module $M$ was introduced by Vasconcelos. It is defined recursively on the dimension as follows:

Definition 5.1 ([Va, Definition 9.4.1]). The homological degree of $M$ is the number

$$
\operatorname{hdeg}(M)=\operatorname{deg}(M)+\sum_{i=0}^{d-1}\left(\begin{array}{c}
d-1 \\
i
\end{array}\right) \operatorname{hdeg}\left(\operatorname{Ext}_{R}^{n+i+1-d}(M, R)\right) .
$$

Note that

(a) $\operatorname{hdeg}(M) \geq \operatorname{deg}(M)$, and the equality holds if and only if $M$ is a CohenMacaulay module.

(b) $\operatorname{hdeg}(M)=\operatorname{hdeg}\left(M / H_{\mathfrak{m}}^{0}(M)\right)+\operatorname{dim}_{k}\left(H_{\mathfrak{m}}^{0}(M)\right)$.

Let gen $(M)$ denote the maximal degree of elements in a minimal set of homogeneous generators of $M$. It turns out that the homological degree gives an upper bound for the Castelnuovo-Mumford regularity

$$
\operatorname{reg}(M) \leq \operatorname{gen}(M)+\operatorname{hdeg}(M)-1 .
$$

This result was first proved for rings by Doering, Gunston and Vasconcelos (DGV), Theorem 2.4]). Later on it was extended to modules by Nagel ([Na, Theorem 3.1]). 
It was also shown in $\mathrm{HHy} 2$ that one can use $\operatorname{hdeg}(M)$ to bound the CastelnuovoMumford regularity of Ext modules. In Chapter 9 of the book Va] one can find some interesting applications of this invariant. Therefore Vasconcelos asked the following question (see the last two lines on page 261 of $[\mathrm{Va}]$ ):

Is the homological degree bounded by a polynomial function of the CastelnuovoMumford regularity?

The following result gives a positive answer to this question.

Theorem 5.2. Let $M$ be a non-zero finitely generated graded $R$-module of dimension $d>0$. Then

$$
\operatorname{hdeg}(M) \leq\left[\mu(M)\left(\begin{array}{c}
\operatorname{reg}(M)-\operatorname{indeg}(M)+n \\
n
\end{array}\right)\right]^{2^{(d-1)^{2}}} .
$$

In order to prove this theorem we need some auxiliary results.

Lemma 5.3. (i) $\operatorname{deg}(M)+\operatorname{dim}_{k} H_{\mathfrak{m}}^{0}(M) \leq \sum_{\mu=\operatorname{indeg}(M)}^{\operatorname{reg}(M)} H_{M}(\mu)$.

(ii) $\sum_{\mu=\operatorname{indeg}(M)}^{\mathrm{reg}(M)} H_{M}(\mu) \leq \mu(M)(\underset{n}{\operatorname{reg}(M)-\operatorname{indeg}(M)+n})$.

Proof. (i) Let $l_{1}, \ldots, l_{d}$ be a generic linear s.o.p. of $M$ and $\bar{M}=M / H_{\mathfrak{m}}^{0}(M)$. Note that $\operatorname{reg}\left(\bar{M} /\left(l_{1}, \ldots, l_{d}\right) \bar{M}\right) \leq \operatorname{reg}(\bar{M}) \leq \operatorname{reg}(M)$ and $\operatorname{indeg}\left(\bar{M} /\left(l_{1}, \ldots, l_{d}\right) \bar{M}\right) \geq$ $\operatorname{indeg}(\bar{M}) \geq \operatorname{indeg}(M)$. Hence

$$
\begin{aligned}
\operatorname{deg}(M) & =\operatorname{deg}(\bar{M}) \leq \operatorname{dim}_{k}\left(\bar{M} /\left(l_{1}, \ldots, l_{d}\right) \bar{M}\right) \\
& =\sum_{\mu=\operatorname{indeg}(M)}^{\operatorname{reg}(M)} \operatorname{dim}_{k}\left[\bar{M} /\left(l_{1}, \ldots, l_{d}\right) \bar{M}\right]_{\mu} \leq \sum_{\mu=\operatorname{indeg}(M)}^{\operatorname{reg}(M)} \operatorname{dim}_{k}\left(\bar{M}_{\mu}\right) .
\end{aligned}
$$

On the other hand, $H_{\mathfrak{m}}^{0}(M)_{\mu}=0$ for all $\mu<\operatorname{indeg}(M)$ and $\mu>\operatorname{reg}(M)$. This yields

$$
\begin{aligned}
\operatorname{deg}(M)+\operatorname{dim}_{k} H_{\mathfrak{m}}^{0}(M) & \leq \sum_{\mu=\operatorname{indeg}(M)}^{\operatorname{reg}(M)}\left[\operatorname{dim}_{k}\left(\bar{M}_{\mu}\right)+\operatorname{dim}_{k} H_{\mathfrak{m}}^{0}(M)_{\mu}\right] \\
& =\sum_{\mu=\operatorname{indeg}(M)}^{\operatorname{reg}(M)} H_{M}(\mu) .
\end{aligned}
$$

(ii) We may assume that $\operatorname{indeg}(M)=0$. Then the inequality follows from Lemma 4.4 (ii).

Lemma 5.4. Let $\bar{M}=M / H_{\mathfrak{m}}^{0}(M)$ and $\bar{r}=\operatorname{reg}(\bar{M})$.

(i) If $d \leq 1$, then $\operatorname{hdeg}(M)=\operatorname{dim}_{k}\left(H_{\mathfrak{m}}^{0}(M)\right)+H_{\bar{M}}(\bar{r})$.

(ii) If $d \geq 2$, then $\operatorname{hdeg}\left(\operatorname{Ext}_{R}^{n-1}(M, R)\right) \leq\left(H_{\bar{M}}(\bar{r})-\operatorname{deg}(M)\right) H_{\bar{M}}(\bar{r})$.

Proof. (i) The statement is trivial for $d=0$.

If $d=1$, then by (5.1) $\operatorname{hdeg}(M)=\operatorname{deg}(\bar{M})+\operatorname{dim}_{k}\left(H_{\mathfrak{m}}^{0}(M)\right)$. Since $\operatorname{dim}(\bar{M})=1$, by Lemma 4.1(i) $\operatorname{deg}(\bar{M})=P_{\bar{M}}(\bar{r})=H_{\bar{M}}(\bar{r})$. Hence hdeg $(M)=\operatorname{dim}_{k}\left(H_{\mathfrak{m}}^{0}(M)\right)+$ $H_{\bar{M}}(\bar{r})$.

(ii) Let $d \geq 2$. Without loss of generality we may assume that $M=\bar{M}$, i.e. $\operatorname{depth}(M)>0$, and hence $r:=\operatorname{reg}(M)=\bar{r}$. For simplicity, let $E_{1}=\operatorname{Ext}_{R}^{n-1}(M, R)$. Since $\operatorname{dim}\left(E_{1}\right) \leq 1$ (see [Sc, p. 63]), by (5.1)

$$
\operatorname{hdeg}\left(E_{1}\right)=\operatorname{dim}_{k}\left(H_{\mathfrak{m}}^{0}\left(E_{1}\right)\right)+\operatorname{deg}\left(E_{1}\right) .
$$


Let $M^{\prime}=M_{\geq r}$ and $E_{1}^{\prime}:=\operatorname{Ext}_{R}^{n-1}\left(M^{\prime}, R\right)$. Using the exact sequence (3.1) we get $\operatorname{dim}_{k}\left(H_{\mathfrak{m}}^{0}\left(E_{1}\right)\right) \leq \operatorname{dim}_{k}\left(H_{\mathfrak{m}}^{0}\left(E_{1}^{\prime}\right)\right)$ and $\operatorname{deg}\left(E_{1}\right)=\operatorname{deg}\left(E_{1}^{\prime}\right)$. Hence, by Lemma 5.3(i) we get

$$
\operatorname{hdeg}\left(E_{1}\right) \leq \operatorname{dim}_{k}\left(H_{\mathfrak{m}}^{0}\left(E_{1}^{\prime}\right)\right)+\operatorname{deg}\left(E_{1}^{\prime}\right) \leq \sum_{\mu=\operatorname{nndeg}\left(E_{1}^{\prime}\right)}^{\operatorname{reg}\left(E_{1}^{\prime}\right)} \operatorname{dim}_{k}\left(\left(E_{1}^{\prime}\right)_{\mu}\right) .
$$

Since $\operatorname{depth}\left(M^{\prime}\right)>0$ and $\operatorname{reg}\left(M^{\prime}\right)=r$, by Theorem 3.5

$$
\operatorname{reg}\left(E_{1}^{\prime}\right)+n-1 \leq \max \left\{P_{M^{\prime}}(r-1)-r,-r-1\right\} .
$$

Let $y$ be a generic linear form. Note that $r \leq \operatorname{indeg}\left(M^{\prime} / y M^{\prime}\right) \leq \operatorname{reg}\left(M^{\prime} / y M^{\prime}\right) \leq$ $\operatorname{reg}\left(M^{\prime}\right)=r$. This implies that $M^{\prime} / y M^{\prime}$ is generated in degree $r$ and that by Lemma 4.1(ii)

$$
H_{\overline{M^{\prime} / y M^{\prime}}}(r) \geq \operatorname{deg}\left(M^{\prime} / y M^{\prime}\right)=\operatorname{deg}(M) .
$$

Therefore, by Lemma 4.1(i) we get

$$
\begin{aligned}
P_{M^{\prime}}(r-1) & =P_{M^{\prime}}(r)-P_{M^{\prime} / y M^{\prime}}(r)=H_{M^{\prime}}(r)-H_{\overline{M^{\prime} / y M^{\prime}}}(r) \\
& \leq H_{M}(r)-\operatorname{deg}(M) .
\end{aligned}
$$

This yields

$$
\operatorname{reg}\left(E_{1}^{\prime}\right)+n-1 \leq \max \left\{H_{M}(r)-\operatorname{deg}(M)-r,-r-1\right\} \leq H_{M}(r)-r-1 .
$$

Thus

By Theorem 2.4(1),

$$
\operatorname{reg}\left(E_{1}^{\prime}\right) \leq H_{M}(r)-r-n
$$

$$
\operatorname{indeg}\left(E_{1}^{\prime}\right) \geq-r-n+1
$$

By Theorem 4.2 and the inequality (5.3),

$$
\operatorname{dim}_{k}\left(\left(E_{1}^{\prime}\right)_{\mu}\right) \leq P_{M}(r-1) \leq H_{M}(r)-\operatorname{deg}(M)
$$

for all $\mu$. Hence, by (5.2) we finally obtain $\operatorname{hdeg}\left(E_{1}\right) \leq\left(\operatorname{reg}\left(E_{1}^{\prime}\right)-\operatorname{indeg}\left(E_{1}^{\prime}\right)+1\right)\left(H_{M}(r)-\operatorname{deg}(M)\right) \leq\left(H_{M}(r)-\operatorname{deg}(M)\right) H_{M}(r)$, as required.

The following result gives a bound on the cohomological degree in terms of the Hilbert polynomial.

Theorem 5.5. Let $M$ be a non-zero finitely generated graded $R$-module of dimension $d \geq 1$. Let $\bar{M}=M / H_{\mathfrak{m}}^{0}(M)$ and $\bar{r}=\operatorname{reg}(\bar{M})$. Then

$$
\operatorname{hdeg}(M) \leq \operatorname{dim}_{k}\left(H_{\mathfrak{m}}^{0}(M)\right)+\left(P_{M}(\bar{r})\right)^{2^{(d-1)^{2}}} .
$$

Proof. By Lemma 4.1(i) it is equivalent to prove that

$$
\operatorname{hdeg}(M) \leq \operatorname{dim}_{k}\left(H_{\mathfrak{m}}^{0}(M)\right)+\left(H_{\bar{M}}(\bar{r})\right)^{2^{(d-1)^{2}}} .
$$

We do induction on $d$. For simplicity we set $E_{j}:=\operatorname{Ext}_{R}^{n-j}(M, R)$ and $H:=H_{\bar{M}}(\bar{r})$.

The case $d=1$ was proved in Lemma 5.4 .

Let $d=2$. Then, by Lemma 5.4 we get

$$
\begin{aligned}
\operatorname{hdeg}(M) & =\operatorname{dim}_{k}\left(H_{\mathfrak{m}}^{0}(M)\right)+\operatorname{deg}(M)+\operatorname{hdeg}\left(E_{1}\right) \\
& \leq \operatorname{dim}_{k}\left(H_{\mathfrak{m}}^{0}(M)\right)+\operatorname{deg}(M)+(H-\operatorname{deg}(M)) H \leq \operatorname{dim}_{k}\left(H_{\mathfrak{m}}^{0}(M)\right)+H^{2} .
\end{aligned}
$$


Let $d \geq 3$. If $H=1$, then from the exact sequence

$$
\bar{M}_{\bar{r}-1} \rightarrow \bar{M}_{\bar{r}} \rightarrow(\bar{M} / y \bar{M})_{\bar{r}} \rightarrow 0,
$$

where $y$ is a generic linear form, and $(\bar{M} / y \bar{M})_{\bar{r}} \neq 0$ (since $\operatorname{dim}(\bar{M} / y \bar{M})>0$ and $\bar{M} / y \bar{M}$ is generated in degrees at most $\bar{r})$ we get that $\bar{M} \cong R / I(-\bar{r})$ for some homogeneous ideal $I$ and $\operatorname{reg}(R / I)=0$. Hence $I$ is generated by linear forms and $\bar{M}$ is a Cohen-Macaulay module. In this case, by (5.1)

$$
\operatorname{hdeg}(M)=\operatorname{dim}_{k}\left(H_{\mathfrak{m}}^{0}(M)\right)+\operatorname{deg}(M)=\operatorname{dim}_{k}\left(H_{\mathfrak{m}}^{0}(M)\right)+1,
$$

and the above required inequality trivially holds.

From now on we assume that $H \geq 2$. Fix an $i$ such that $2 \leq i \leq d-1$. In the sequel we want to bound $\operatorname{hdeg}\left(E_{i}\right)$. Hence, for this part we may assume that $\operatorname{depth}(M)>0$, and so $r:=\operatorname{reg}(M)=\bar{r}$.

By Theorem 3.5 and Lemma 4.1(i),

$$
\operatorname{reg}\left(E_{i}\right) \leq\left(C_{d, d-i} H\right)^{2^{d-2}}-\bar{r}+1-n+i,
$$

where $C_{d, j}=\max \left\{\left(\begin{array}{c}d-1 \\ j\end{array}\right),\left(\begin{array}{c}d-1 \\ j+1\end{array}\right)\right\}$. Note that $\sum_{2 j \leq d-1}\left(\begin{array}{c}d-1 \\ 2 j\end{array}\right)=\sum_{2 j+1 \leq d-1}\left(\begin{array}{c}d-1 \\ 2 j+1\end{array}\right)=$ $2^{d-2}$. Therefore $C_{d, j} \leq 2^{d-2}-1$ for all $j$ and $d \geq 3$. Since $H \geq 2$, this implies

$$
C_{d, d-i} H \leq\left(2^{d-2}-1\right) H \leq H^{d-1}-2 .
$$

Hence

$$
\operatorname{reg}\left(E_{i}\right) \leq\left(H^{d-1}-2\right)^{2^{d-2}}-\bar{r}+1-n+i .
$$

Using Corollary 4.3, we see that the following holds for all $\mu \leq \operatorname{reg}\left(E_{i}\right)$ :

$$
H_{E_{i}}(\mu) \leq\left(\begin{array}{c}
\left(H^{d-1}-2\right)^{2^{d-2}}+i \\
i-1
\end{array}\right) H \leq\left(\begin{array}{c}
\left(H^{d-1}-2\right)^{2^{d-2}}+d-1 \\
d-2
\end{array}\right) H .
$$

Again using the inequality $\left(\begin{array}{c}a+\delta \\ \delta\end{array}\right)<(a+1)^{\delta}$ for all $a$ and $\delta \geq 1$, from (5.5) we get

$$
\begin{aligned}
H_{E_{i}}\left(\operatorname{reg}\left(E_{i}\right)\right) & \leq H\left(\left(H^{d-1}-2\right)^{2^{d-2}}+2\right)^{d-2} \\
& \leq H\left(H^{(d-1) 2^{d-2}}-2 \cdot 2^{d-2}+2\right)^{d-2} \\
& <H^{(d-1)(d-2) 2^{d-2}+1} .
\end{aligned}
$$

By induction on $d$ it is easy to check that $(d-2)(d-1) 2^{d-2}+1<2^{2 d-3}-2$ for all $d \geq 3$. Hence, the above inequality yields

$$
H_{E_{i}}\left(\operatorname{reg}\left(E_{i}\right)\right)<H^{2^{2 d-3}-2} .
$$

On the other hand, by Theorem 2.4(1), indeg $\left(E_{i}\right) \geq-\bar{r}-n+i$. Using Lemma 5.3(i) together with (5.4) and (5.6), we have

$$
\begin{aligned}
\operatorname{dim}_{k}\left(H_{\mathfrak{m}}^{0}\left(E_{i}\right)\right) & <\left(\operatorname{reg}\left(E_{i}\right)-\operatorname{indeg}\left(E_{i}\right)+1\right) H^{(d-1)(d-2) 2^{d-2}+1} \\
& \leq H^{(d-1) 2^{d-2}} H^{(d-1)(d-2) 2^{d-2}+1} \\
& <H^{2\left((d-1)(d-2) 2^{d-2}+1\right)} \\
& <H^{2\left(2^{2 d-3}-2\right)}
\end{aligned}
$$


Since $\operatorname{dim} E_{i} \leq d-1$ (see [Sc, p. 63]), by the induction hypothesis, (5.7) and (5.8) we get

$$
\begin{aligned}
\operatorname{hdeg}\left(E_{i}\right) & <H^{2\left(2^{2 d-3}-2\right)}+\left(H^{2^{2 d-3}-2}\right)^{2^{(d-2)^{2}}} \\
& \leq 2 H^{2^{(d-1)^{2}}-2^{(d-2)^{2}+1}} \\
& \leq 2 \frac{H^{2^{(d-1)^{2}}}}{2^{2^{(d-2)^{2}+1}}}
\end{aligned}
$$

Now we are ready to estimate $\operatorname{hdeg}(M)$. Using (5.1), (5.8), (5.9) and Lemma 5.4. we finally get

$$
\begin{aligned}
\operatorname{hdeg}(M) & =\operatorname{deg}(M)+\operatorname{dim}_{k}\left(H_{\mathfrak{m}}^{0}(M)\right)+\operatorname{hdeg}\left(E_{1}\right)+\sum_{i=2}^{d-1}\left(\begin{array}{c}
d-1 \\
i
\end{array}\right) \operatorname{hdeg}\left(E_{i}\right) \\
& <\operatorname{dim}_{k}\left(H_{\mathfrak{m}}^{0}(M)\right)+\operatorname{deg}(M)+(H-\operatorname{deg}(M)) H+\sum_{i=2}^{d-1}\left(\begin{array}{c}
d-1 \\
i
\end{array}\right) 2 \frac{H^{2^{(d-1)^{2}}}}{2^{2^{(d-2)^{2}+1}}} \\
& <\operatorname{dim}_{k}\left(H_{\mathfrak{m}}^{0}(M)\right)+2^{d} \frac{H^{2^{(d-1)^{2}}}}{2^{2^{(d-2)^{2}+1}}} \\
& <\operatorname{dim}_{k}\left(H_{\mathfrak{m}}^{0}(M)\right)+H^{2^{(d-1)^{2}}}
\end{aligned}
$$

In the last estimation we have used the obvious inequality $2^{(d-2)^{2}+1}>d$ for all $d \geq 3$.

Now we can prove Theorem 5.2 as follows:

Proof of Theorem 5.2. Set $\vdots=\operatorname{reg}(M)-\operatorname{indeg}(M)$. If $=0$, then $r=\bar{r}, \mu(M)=$ $H_{M}(r)=H_{\bar{M}}(\bar{r})=P_{M}(\bar{r})+\operatorname{dim}_{k}\left(H_{\mathfrak{m}}^{0}(M)\right)$, and the result follows from Theorem 5.5. If $\iota 0$, by Theorem 5.5. Lemma 5.3 and Lemma 4.4 we have

$$
\begin{aligned}
\operatorname{hdeg}(M) & \leq \mu(M)\left(\begin{array}{c}
+n \\
n
\end{array}\right)+\left[\mu(M)\left(\begin{array}{c}
+n-1 \\
n-1
\end{array}\right)\right]^{2^{(d-1)^{2}}} \\
& \leq \mu(M)\left(\begin{array}{c}
+n \\
n
\end{array}\right)+\left[\mu(M)\left(\begin{array}{c}
+n \\
n
\end{array}\right)-1\right]^{2^{(d-1)^{2}}} \\
& \leq\left[\mu(M)\left(\begin{array}{c}
+n \\
n
\end{array}\right)\right]^{2^{(d-1)^{2}}}
\end{aligned}
$$

as required.

As an immediate consequence of Theorem 5.2 we obtain

Corollary 5.6. Let I be a homogeneous ideal of $R$. Then

$$
\operatorname{hdeg}(R / I) \leq\left[\left(\begin{array}{c}
\operatorname{reg}(R / I)+n \\
n
\end{array}\right)\right]^{2^{(d-1)^{2}}} .
$$




\section{REFERENCES}

[CD] M. Chardin, K. Divaani-Aazar, Generalized local cohomology and regularity of Ext modules, J. Algebra 319 (2008), 4780-4797. MR2416743 (2009c:13043)

[CFN] M. Chardin, A. L. Fall and U. Nagel, Bounds for the Castelnuovo-Mumford regularity of modules, Math. Z. 258 (2008), 69-80. MR2350034 (2009a:13023)

[DGV] L. R. Doering, T. Gunston and W. V. Vasconcelos, Cohomological degrees and Hilbert functions of graded modules, Amer. J. Math. 120 (1998), no. 3, 493-504. MR.1623400 (99h:13019)

[HaH] D. T. Ha and L. T. Hoa, Castelnuovo-Mumford regularity of some modules, Comm. Algebra 36 (2008), 992-1004. MR2394265 (2009a:13024)

[H] L. T. Hoa, Finiteness of Hilbert functions and bounds for Castelnuovo-Mumford regularity of initial ideals, Trans. Amer.Math. Soc. 360 (2008), 4519-4540. MR2403695 (2009b:13035)

[HHy1] L. T. Hoa and E. Hyry, Castelnuovo-Mumford regularity of initial ideals, J. Symb. Comp. 38 (2004), 1327-1341. MR 2168718 (2007b:13028)

[HHy2] L. T. Hoa and E. Hyry, Castelnuovo-Mumford regularity of canonical and deficiency modules, J. Algebra 305 (2006), 877-900. MR2266858 (2007g:13023)

[Na] U. Nagel, Comparing Castelnuovo-Mumford regularity and extended degree: the boderline cases. Trans. Amer. Math. Soc. 357 (2005), no. 9, 3585-3603. MR2146640 (2006h:13027)

[Sc] P. Schenzel, Dualisierende Komplexe in der lokalen Algebra und Buchsbaum-Ringe, Lecture Notes in Mathematics, 907, Springer-Verlag, Berlin-New York, 1982. MR654151 (83i:13013)

[Va] W. V. Vasconcelos, Computational methods in commutative algebra and algebraic geometry. With chapters by David Eisenbud, Daniel R. Grayson, Jürgen Herzog and Michael Stillman. Algorithms and Computation in Mathematics, 2, Springer-Verlag, Berlin, 1998. MR:1484973 (99c:13048)

Institut de Mathématiques de Jussieu, Université Pierre et Marie Curie, 4, Place Jussieu, F-75005 PARis, France

E-mail address: chardin@math.jussieu.fr

Department of Mathematics, University of Vinh, Vietnam

E-mail address: thahanh@yahoo.com

Institute of Mathematics, 18 Hoang Quoc Viet Road, 10307 Hanoi, Vietnam

E-mail address: 1thoa@math.ac.vn 